\title{
Mechanisms of junctional tachycardia showing ventricular pre-excitation
}

\author{
DAVID E WARD, * DAVID H BENNETT, $†$ JOHN CAMM‡
}

From the Departments of Cardiology, ${ }^{\star} S t$ George’s Hospital, London; †Wythenshawe Hospital, Manchester; and $¥ S t$ Bartholomew's Hospital, London

SUMMARY Over a period of five years 12 patients underwent electrophysiological studies for the investigation of recurrent tachycardias which showed ventricular pre-excitation. Nine patients had a type B pattern and two a type A. One patient had episodes of both types. Dual atrioventricular nodal pathways were found in six of seven patients with atrioventricular nodal re-entrant tachycardia mechanisms. Single direct atrioventricular accessory pathways were present in four patients, single nodoventricular pathways in five, and multiple pathways in three. Twenty one tachycardias were induced, of which 13 showed ventricular pre-excitation. Five patients had nodoventricular pathway conduction during atrioventricular nodal tachycardia and one during atrioventricular re-entrant tachycardia. Only three patients had simple antidromic tachycardia and one additional atrioventricular nodal tachycardia with bystander atrioventricular accessory conduction. Three patients had three different tachycardias, three had two types, and six had one type.

Thus junctional tachycardias showing ventricular pre-excitation are often associated with multiple mechanisms and complex anatomical and functional substrates. An accessory pathway was an essential component in only six of 13 tachycardias showing ventricular pre-excitation. Determination of the tachycardia mechanism requires detailed study and analysis.

Several mechanisms of tachycardia have been demonstrated in patients with the Wolff-Parkinson-White syndrome. ${ }^{1}$ By far the commonest mechanism is atrioventricular re-entrant tachycardia with retrograde conduction over the accessory atrioventricular connexion and anterograde conduction over the atrioventricular nodal-His bundle pathway. It is well known, however, that tachycardia may also be caused by re-entrant activity in the reverse direction producing a tachycardia with broad "fully pre-excited" QRS complexes. Furthermore, in the presence of more than one accessory connexion further possibilities exist for the generation of tachycardias with broad QRS complexes not caused by bundle branch block. These tachycardias may cause considerable diagnostic confusion and therapeutic difficulty. Several questions may be defined: $(a)$ the mechanism of tachycardia; $(b)$ the location and type of the accessory pathway; and $(c)$ the functional relation of the pathway to

Requests for reprints to Dr D E Ward, Cardiology Department, St George's Hospital, London SW17 0QT.

Accepted for publication 5 June 1984 the tachycardia circuit. In this report we describe diverse mechanisms of pre-excited tachycardia in 12 patients and how these mechanisms can be elucidated and distinguished using electrophysiological techniques and sometimes antiarrhythmic drugs with specific effects.

\section{Patients and methods}

Twelve patients aged 7-62 (median 20) years were referred for investigation of frequent ( $>10$ a year) recurrent tachycardias associated with ventricular pre-excitation. The Table summarises the relevant details. Electrophysiological studies were performed in all patients as described previously. ${ }^{2}$ In addition to right heart sites, recordings were obtained from the left atrium or coronary sinus in all but three patients (cases 1,7 , and 8). No patient had taken any antiarrhythmic drug for 72 hours before the procedure. None of the patients had received amiodarone. The diagnosis of the cause of ventricular pre-excitation was made according to previously described criteria. ${ }^{34}$ Re-entry junctional tachycardia is defined 
Table Clinical and electrophysiological data

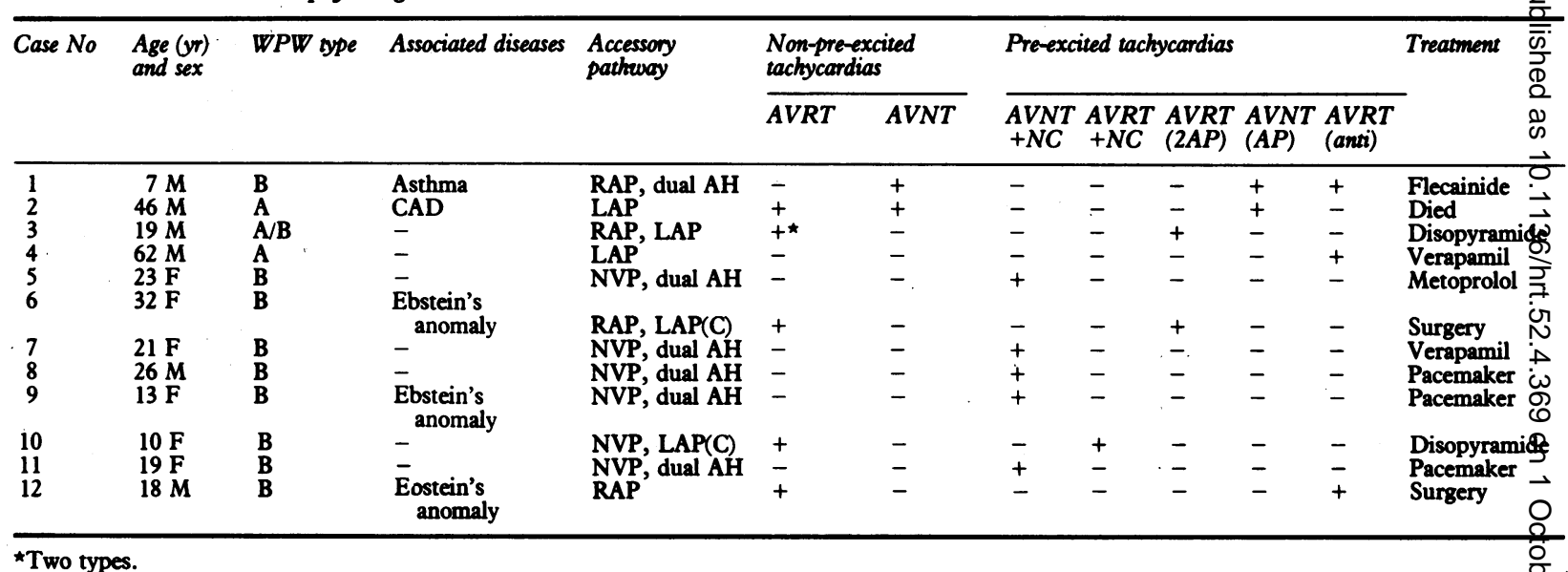

+, present -, absent; WPW, Wolff-Parkinson-White; CAD, coronary artery disease; RAP (LAP), right (left) accessory atrioventricular pathway; N\$P,

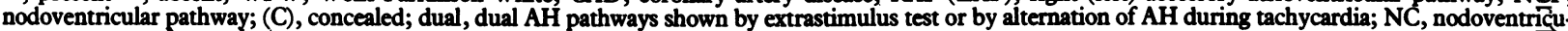

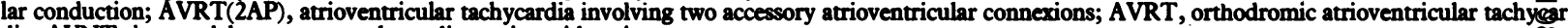
dia; AVNT, intranodal re-entrant tachycardia; anti, antidromic.

as tachycardia due to circus movement using pathways connecting atrial and ventricular myocardium - that is, atrioventricular node-His pathway, direct atrioventricular accessory connexion.

\section{Results}

The Table summarises the individual results. The electrophysiological studies indicated the cause of ventricular pre-excitation in all patients (Table). During the study 21 tachycardias were induced in 12 patients. Three patients had three different tachycardias, three had two types, and six had a single mechanism. During 13 tachycardias the QRS complex was broad and resembled ventricular preexcitation during sinus rhythm or atrial pacing. These tachycardias were considered to show ventricular pre-excitation. An accessory pathway supported preexcited tachycardia in only six of 13 instances.

\section{NODOVENTRICULAR PRE-EXCITATION}

In six patients (cases 5 and 7-11) ventricular preexcitation during tachycardia was the result of nodoventricular conduction. In five of these atrioventricular nodal re-entry was the cause of tachycardia. Three patients have been described in detail in previous communications. ${ }^{45}$ The patient in case 5 typifies this type of pre-excited junctional tachycardia. Figures 1 and 2 illustrate the mechanism of tachycardia (see legends for explanation). This tachycardia is almost certainly due to intra-atrioventricular nodal re-entry with incidental nodoventricular conduction. Another patient (case 11) with features of nodoventricular conduction during atrial paced rhythm showed unusual phenomena. During atrial extrastimulation tachycardia was initiated by a sudden increase in the $\mathrm{AH}$ interval independent of a change in the AV interval, although this had shown gradual prolongation as $A_{1}-A_{2}$ was reduced. The $H V$ interval decreased to $-85 \mathrm{~ms}$. During tachycardia the QRS complexes were broad and showed ventricular preexcitation identical to that seen during atrial extrastimulation. During stable tachycardia the relation of the His potential to the QRS complex was variable. Figure 3 shows a sudden change of HV interval during tachycardia. One possible interpretation is illustrated in Fig. 4a. One patient (case 10) with typical features of nodoventricular conduction was found to have a left concealed ventriculoatrial connexion supporting the retrograde limb of a re-entrant atrioventricular re-entrant tachycardia (Fig. 5). The gradual appearance of pre-excitation during sustained tachycardia , confirms the bystander role of the nodoventricular pathway, which is clearly not required to support tachycardia.

\section{ATRIOVENTRICULAR PRE-EXCITATION}

In seven patients ventricular pre-excitation during tachycardia was caused by conduction over a direct accessory pathway. Three patients had simple antidromic tachycardia with anterograde conduction over the accessory pathway and retrograde re-entrant conduction over the atrioventricular node. Verapamil 0.1 $\mathrm{mg} / \mathrm{kg}$ intravenously was given during this type of tachycardia in one patient (case 4). Slowing of tachycardia as a result of an increase in the retrograde conduction time preceded termination by retrograde block after one minute. This information supports involvement of the atrioventricular node in the retro- 
(a)
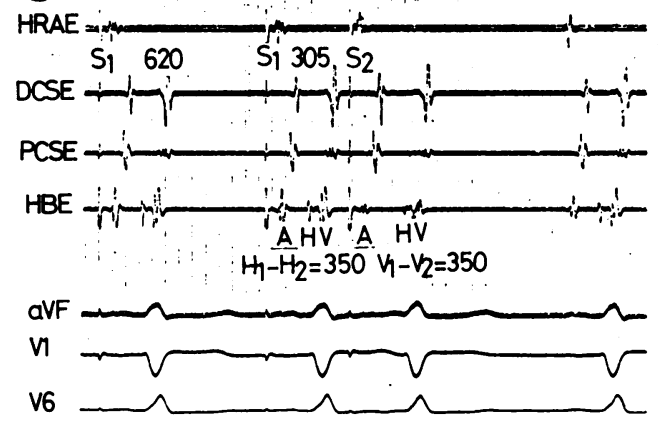

(b)
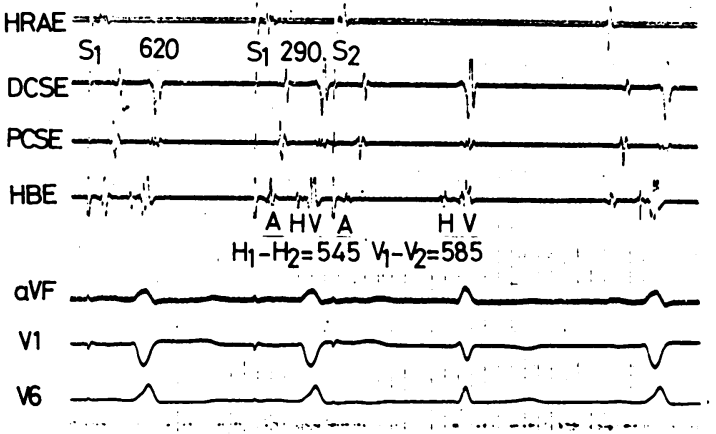

Fig. 1 Case 5: electrograms and electrocardiograms recorded during the atrial extrastimulus method. (a) During regular atrial pacing at a cycle length of $620 \mathrm{~ms}$ the $H V$ interval is zero. Atrial premature stimulation $\left(S_{2}\right)$ results in normal prolongation of the $A H$ interval with no change in the $H V$ interval. (b) An earlier stimulus conducts with a sudden increase in AH interval, normal $H V$ interval (40 ms), and normal QRS. These findings indicate the presence of dual $A H$ pathways and a nodoventricular pathway or fasciculoventricular pathway in close association with the fast pathway. HRAE, high right atrial electrogram; DCSE, distal coronary sinus electrogram; PCSE, proximal coronary sinus electrogram; $H B E$, His bundle electrogram; $A$ atrial, $H$ His, and $V$ ventricular (electrograms). Paper speed 100 $\mathrm{mm} / \mathrm{s}$. (See also Fig. 2.)

grade limb of tachycardia. One of these patients (case 1) had several additional forms of tachycardia. Narrow complex tachycardias of two distinct cycle lengths were observed. Different AH intervals accounted for the differing cycle lengths. On some occasions partial pre-excitation was observed during these tachycardias. Disappearance of partial pre-excitation was associated with an increase in rate due to shortening of the AH interval. Conversion of a narrow or partially pre-excited QRS complex tachycardia to a broad complex tachycardia could be achieved with single ventricular premature stimuli (Fig. 6a). The QRS morphology during this broad complex tachycardia

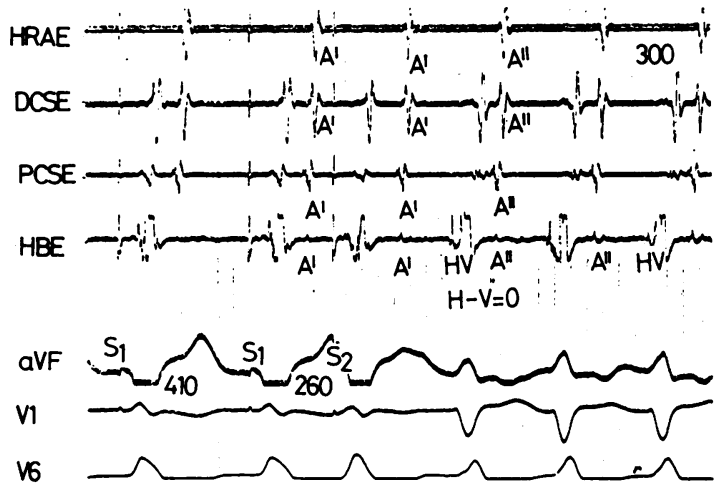

Fig. 2 Case 5: electrograms and electrocardiograms showing initiation of broad complex tachycardia by ventricular extrastimulation. The extrastimulus $\left(S_{2}\right)$ conducts to the atria via the atrioventricular node. Tachycardia shows an $\mathrm{HV}$ interval of zero and a QRS complex identical to that seen during atrial rhythms (see Fig. 1). A circuit comprising the nodoventricular pathway anterogradely with retrograde conduction to the His bundle (a subjunctional tachycardia) is most unlikely to account for these findings. The evidence supports atrioventricular nodal re-entry with an unusually long $H A$ interval. Conduction over the nodoventricular pathway is incidental. There was no evidence of a direct atrioventricular pathway. Retrograde conduction studies were normal. Abbreviations as in Fig. 1 .

suggested right ventricular pre-excitation. The uniformity of the tachycardia cycle length regardless of the cycle length of the preceding narrow tachycardia suggested a different mechanism (Fig. 6b). Although narrow complex tachycardias could not be terminated by atrial underdrive pacing, broad complex tachycardias were easily terminated with this method. Verapamil $0.1 \mathrm{mg} / \mathrm{kg}$ given intravenously as a bolus during the wide complex tachycardia had no effect. In contrast, flecainide ( $2 \mathrm{mg} / \mathrm{kg}$ ) given over five minutes terminated tachycardia in the anterograde limb. These data strongly suggest the presence of a direct atrioventricular accessory connexion supporting the anterograde limb of fully pre-excited tachycardia. This pathway probably acted as a bystander during the narrow QRS tachycardias of intranodal origin. In both types of tachycardia the retrograde sequence was identical, with early low right atrial activation. Alternatively, a second concealed accessory pathway may have been the retrograde limb of all tachycardias. This would explain the total absence of any effect of verapamil, which may terminate simple antidromic tachycardias in the retrograde limb as in the patient in case 4 . Atrioventricular nodal tachycardia with incidental conduction over a direct right sided accessory pathway occurred in one other patient. This patient also had orthod- 


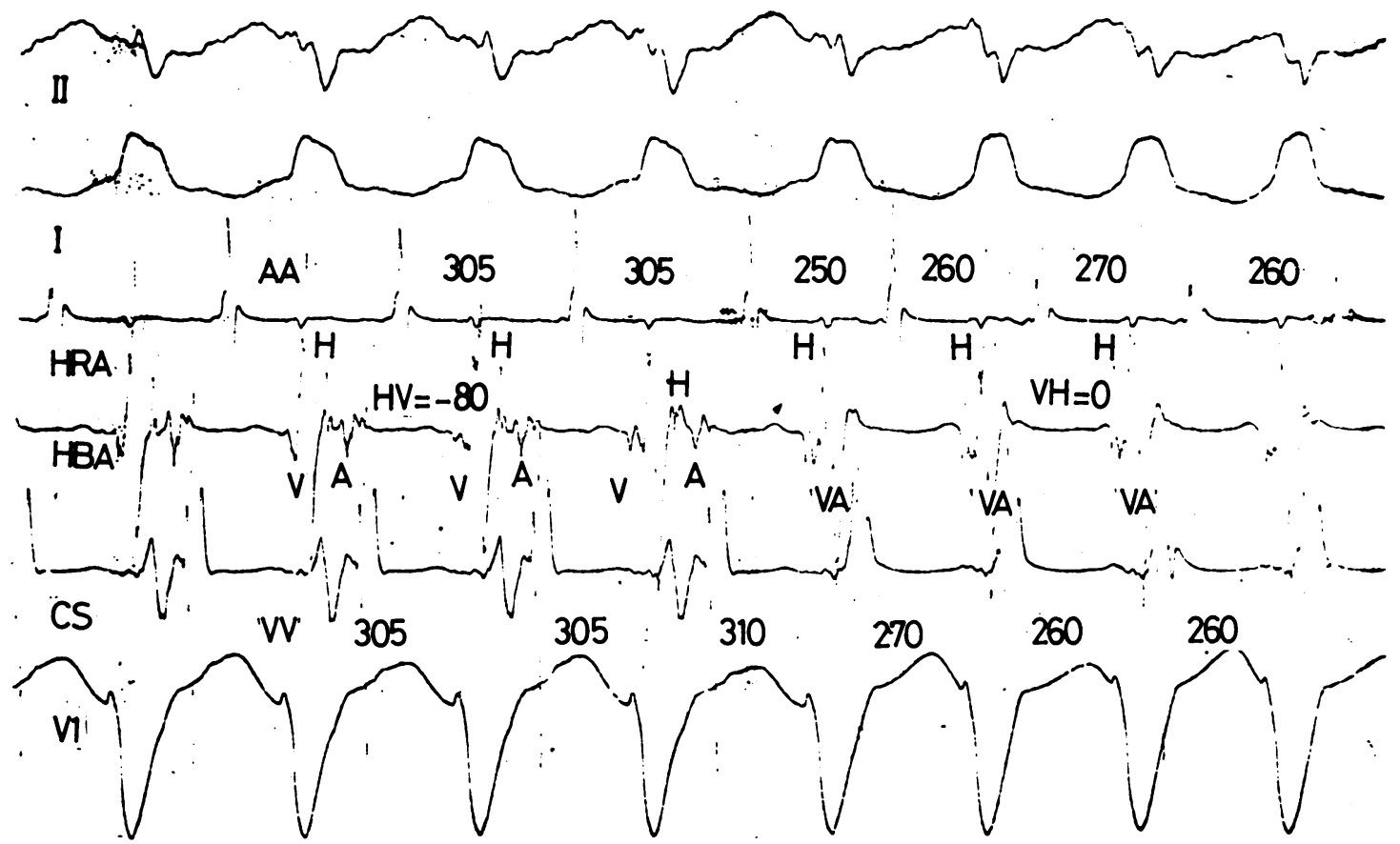

Fig. 3 Case 11: electrograms and electrocardiograms during stable tachycardia. The recordings to the left show tachycardia with a stable cycle length of $305 \mathrm{~ms}$. HV interval $-80 \mathrm{~ms}$. Suddenly the His potential appears before the ventricular electrogram with an HV interval of zero. This event is followed by a shortening of the ventriculoatrial interval, atrial cycle length, and subsequently the ventricular cycle length, and tachycardia again stabilises. The HHRA interval increases transiently immediately after the shortening of the $V H$ interval but is not changed during faster tachycardia. The AV interval has increased from 115 to 145 ms. This excludes a direct atrioventricular connexion as a bystander or involvement of a direct atrioventricular connexion in the anterograde limb. On other occasions a transient lesser shortening of the VH interval and cycle length was seen. (Fig. 4 shows the postulated mechanism of these changes.)

romic atrioventricular re-entrant tachycardia (Fig. 7). Two patients (cases 3 and 6) had tachycardias which did not involve the atrioventricular node. In both patients anterograde conduction occurred over a right accessory pathway and retrograde conduction over a left concealed accessory pathway. In one patient this situation was transient. In the other (case 3) intravenous verapamil $(0.1 \mathrm{mg} / \mathrm{kg})$ had no effect on this type of tachycardia, although it terminated the typical orthodromic variety. On another occasion, intravenous flecainide readily terminated tachycardia involving two accessory pathways.

The Table shows the final treatment of each patient. One patient (case 6) underwent surgical ablation of the septal accessory atrioventricular pathway and His bundle. The right sided accessory pathway could not be ablated. Tachycardias have not recurred. In the patient in case 12 the right accessory pathway was successfully located and ablated. Three patients had implanted antitachycardia pacemakers, and in the remainder the tachycardias were controlled with antiarrhythmic drugs.

\section{Discussion}

The most frequent cause of wide complex tachycardia in the Wolff-Parkinson-White syndrome is atrioventricular re-entrant tachycardia with rate related bundle branch delay or block. ${ }^{6}$ In this analysis we have not considered these tachycardias but rather those in which the wide QRS complex during tachycardia was accounted for by conduction over an accessory pathway resulting in ventricular pre-excitation. This may occur in one of two ways: $(a)$ the accessory connexion is part of the tachycardia circuit or $(b)$ it is activated incidentally by tachycardia occurring as a result of some independent mechanism. The most usual cause of the phenomenon is the first, in which activation proceeds from atria to ventricles over an accessory pathway and returns via the atrioventricular node or 


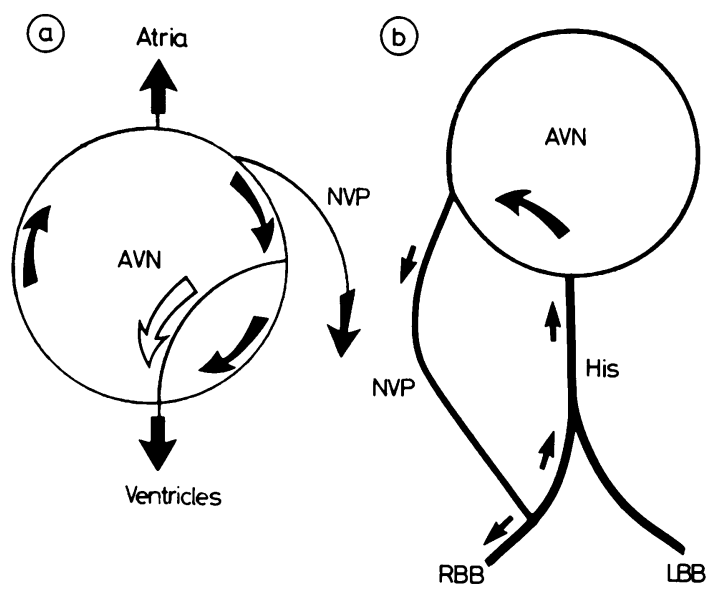

Fig. 4 Diagrams of postulated mechanisms of tachycardia in Fig. 3. (a) The initial event is a change in the relative timing of the His potential independent of a change in the ventricular cycle. The sudden advancement of the His potential must therefore have occurred after engagement of the nodoventricular pathway (NVP). A faster intranodal pathway (open arrow) distal to the accessory pathway accounts for the shorter $H V$ interval, the earlier timing of the next atrial electrogram and the subsequent $Q R S$ complex, and the shorter tachycardia cycle length. As the His bundle is not on circuit in this mechanism changes in the $H V$ interval are not necessarily exactly reflected by changes in cycle length. In the new stable tachycardia the $A V$ interval has increased by $30 \mathrm{~ms}$ without affecting the $H V$ relation indicating some delay in the anterograde limb proximal to the accessory connexion, which possibly accounts for the difference between $V H$ shortening and tachycardia cycle decrease. (b) A possible mechanism of re-entrant tachycardia involves the nodoventricular pathway. ${ }^{13}$ The nodoventricular pathway may be in close association with the $A V$ node $(A V N)$. Pre-excitation during tachycardia could be due to anterograde conduction over the nodoventricular pathway. This pathway may insert into the myocardium or the right bundle branch. Retrograde conduction via the His-Purkinje system to the atrioventricular node completes a potential circuit. To explain an $H V$ interval of zero the nodoventricular pathway must insert directly into the right bundle branch (as drawn). If more than one retrograde pathway exists (distal His-Purkinje branches) the activation of the His bundle could vary with respect to the onset of the $Q R S$ (varying $H V$ interval). This model, however, does not explain all the features observed in the patient in case 11. RBB (LBB), right (left) bundle branch.

any other available pathway. The exact prevalence of this form of tachycardia is not known. Wellens et al were able to initiate this tachycardia in four of 87 patients undergoing electrophysiological studies. ${ }^{7}$ Benditt et al, in a review of their experience of wide complex pre-excited tachycardias, noted this mechanism in seven of 26 patients. ${ }^{8}$ In three of these patients more than one accessory pathway was demonstrated. Three of our patients had simple antidromic tachycardia (cases 1,4 , and 12), and in a further two anterograde conduction occurred over one accessory connexion and the retrograde limb of the circuit was formed by a second accessory pathway. In one patient the two pathways were capable of anterograde and retrograde conduction, a finding reported only once previously. ${ }^{9}$

Although the combination of dual AH pathways and accessory atrioventricular pathways is well recognised, ${ }^{10}$ there are few reports of intranodal re-entrant tachycardia with incidental conduction over an accessory atrioventricular connexion. Sudden onset and termination of pre-excitation without changes in tachycardia cycle length or atrial sequence are highly suggestive of incidental pre-excitation. ${ }^{11}$ Furthermore, varying degrees of pre-excitation during tachycardia exclude anterograde on-circuit activation of the accessory pathway, which must therefore be a bystander.

The role of nodoventricular pathways in tachycardias has been discussed at length in published reports. ${ }^{11-13}$ The central question is whether or not these pathways support tachycardias or act as bystanders. Gallagher et al $^{13}$ have presented strong arguments that nodoventricular conduction during tachycardia indicates the involvement of this pathway in the anterograde limb of what is essentially a ventricular or subjunctional tachycardia ${ }^{14}$ incorporating accessory and intraventricular conduction pathways. In several instances they showed ventriculoatrial dissociation during tachycardia, which, although supportive of subjunctional macroreentry, does not exclude intranodal re-entry. ${ }^{15}$ The macroreentrant mechanism is undoubtedly possible, but as pointed out by Akhtar $^{6}$ no unequivocal proof as yet exists. Two of our patients illustrate this controversy. The findings in one patient (case 5) favour an intranodal rather than a subjunctional mechanism (Figs. 1 and 2 ). These findings strongly resemble those we have found in other patients. ${ }^{45}$ Another patient (case 11) with features of accessory nodoventricular conduction showed unusual phenomena, which could be interpreted as reflecting either a junctional or subjunctional mechanism of tachycardia. During tachycardia the relation of the His potential to the QRS (HV interval) changed suddenly (Figs. 3, 4, and 5). Kuck et al noticed similar changes during simple antidromic tachycardias which were attributable to equal changes in the VH interval. ${ }^{16}$ Trantham et al have reported three patients with findings similar to those in our patient. ${ }^{17}$ Each case showed tachycardia with either long or short VH intervals. These findings were interpreted as consistent with the hypothesis that the tachycardia circuit comprised anterograde nodoventricular conduction and retrograde His-Purkinje conduction (Fig. 4b). They suggested that changes in intraventricular conduction time accounted for both 


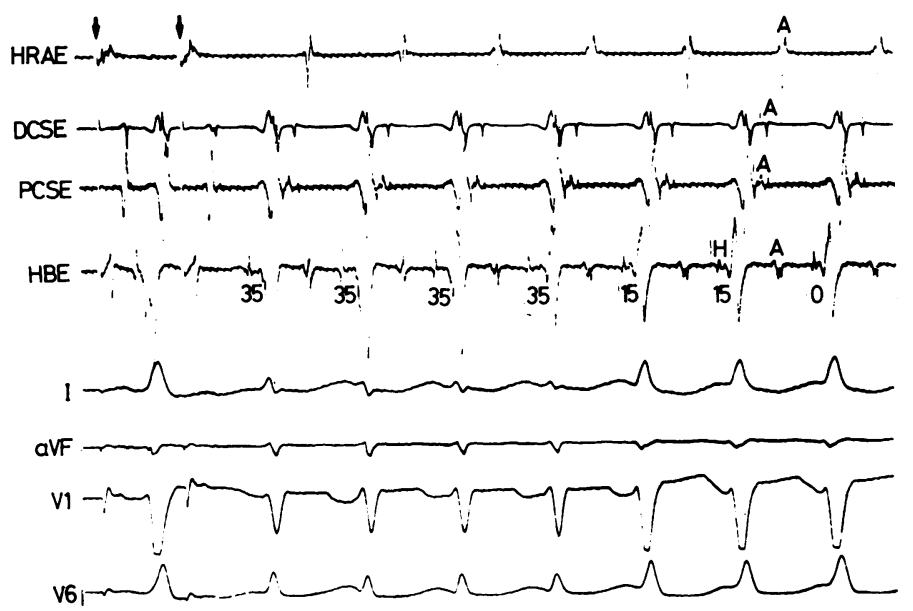

Fig. 5 Case 10: electrograms and electrocardiograms showing the initiation of tachycardia. During regular atrial pacing the $Q R S$ complexes show left bundle branch block morphology and a short $H V$ interval. An atrial extrastimulus conducts with a normal $\mathrm{HV}$ interval ( $35 \mathrm{~ms}$ ) and a normal $Q R S$ complex, and tachycardia is initiated. Earliest atrial activation during tachycardia is seen on the coronary sinus electrogram (PCSE). After several complexes the $H V$ interval shortens because pre-excitation returns. These findings indicate anterograde atrioventricular nodal and retrograde direct accessory ventriculoatrial conduction sustaining tachycardia with incidental nodoventricular conduction independent of the tachycardia mechanism. Abbreviations as in Fig. 1.

(a)

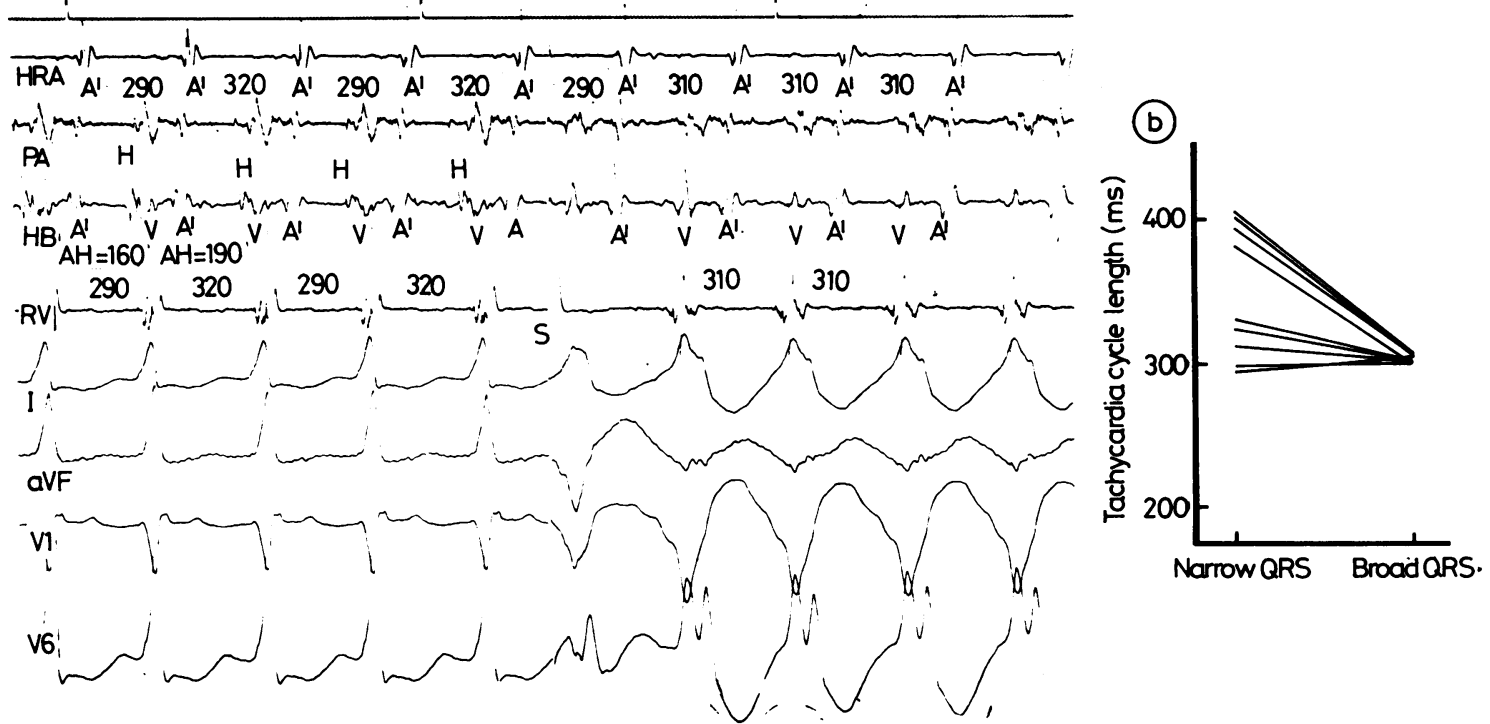

Fig. 6 (a) Case 1; electrograms and electrocardiograms during stable tachycardia. Initially tachycardia shows partial pre-excitation with an HV interval of $25 \mathrm{~ms}$ and cycle length alternation of 290/320 ms. A single right ventricular stimulus results in conversion to tachycardia with fully pre-excited QRS complexes and a stable cycle length of $310 \mathrm{~ms}$. This phenomenon was also observed during slower narrow complex tachycardia. The cycle length of the resulting wide complex tachycardia was always 300-310 ms regardless of the initial cycle length. (b) Diagram showing changes in tachycardia cycle length produced by a single ventricular stimulus. The narrow or partially pre-excited tachycardias fell into slow and fast categories. These tachycardias showed varying degrees of pre-excitation which on occasion could be related to changes in the $A H$ interval. This group of tachycardias was thought to reflect atrioventricular nodal re-entry with variable degrees of incidental pre-excitation over a right accessory pathway. Conversion to wide complex tachycardia was associated with uniformity of cycle length regardless of the initial tachycardia. The retrograde sequence remains unaltered. The wide complex tachycardia was considered to reflect atrioventricular re-entry with anterograde conduction over the right accessory pathway. The retrograde limb is either the normal atrioventricular node or an additional accessory pathway. It is suggested that the ventricular premature stimulus responsible for the conversion conducted retrogradely with consequent earlier anterograde accessory pathway activation and incorporation in the circuit. 


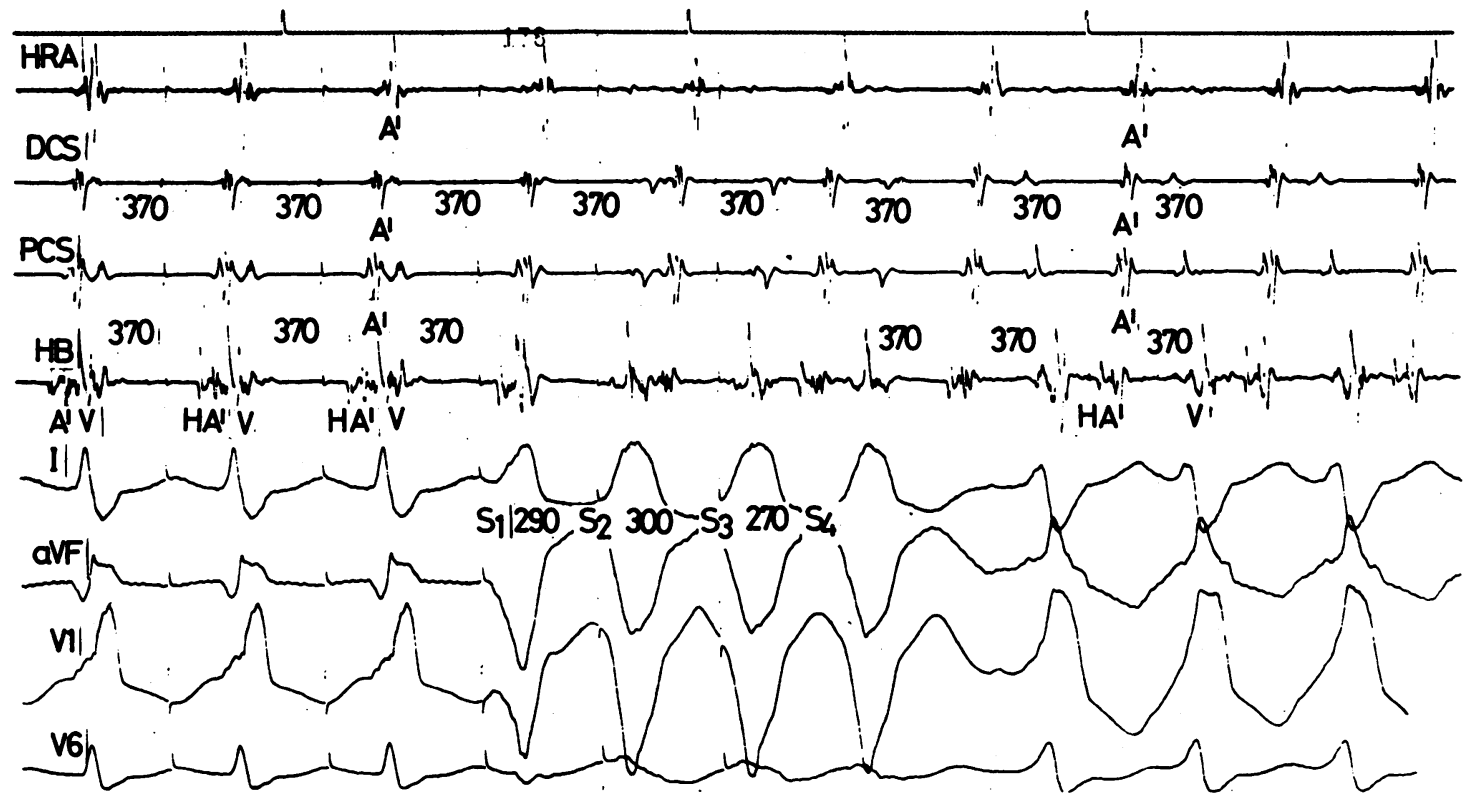

Fig. 7 Case 2: electrograms and electrocardiograms showing initial tachycardia with right bundle branch block (fixed), a normal HV interval, and atrial activation before ventricular activation with a normal retrograde sequence. Four ventricular paced stimuli result in conversion to fully pre-excited tachycardia without disturbing the atrial cycle or the His cycle. The HA relation and the retrograde sequence remain unchanged. Presumably, there is concealed retrograde conduction in the left sided accessory pathway during narrow complex tachycardias. The effect of ventricular pacing may be removal of this concealed conduction by rephasing the refractory time of this pathway allowing anterograde expression during undisturbed intranodal re-entry.

the change in the VH interval and the tachycardia cycle length. It is of interest to note, however, that the change in VH interval in each case exceeds the change in tachycardia cycle length just as in our patient. The increase in cycle length should equal that of the VH interval unless there is consequent delay in another part of the circuit such as the atrioventricular node. Thus shortening of the VH interval may be followed by increasing intranodal delay resulting in a shortening of tachycardia cycle length somewhat less than the change in the VH interval. This delay is most likely to occur in that part of the postulated circuit between the His bundle and the nodoventricular pathway. If this were so, however, a longer HA interval would be expected during faster stable tachycardia reflecting additional retrograde intranodal delay. In our patient this was not seen. To account for the VH interval of zero during tachycardia it would be necessary to postulate insertion of the nodoventricular fibre into the right bundle branch with anterograde conduction time to the myocardium equal to retrograde conduction time to the His bundle. Such a mechanism would also be expected to operate during atrial rhythms if anterograde His activation were sufficiently delayed relative to ventricular activation. Since negative HV values were seen during atrial extrastimulation, this mechanism need not be invoked in this particular instance. Nodoventricular pathways may have complex relations with intranodal structures. For example, such pathways appear to be uniquely functionally associated with one of two intranodal pathways ${ }^{18-20}$ as observed in one of our patients (case 5). The observations in the patient in case 12 may reflect a more complex interrelation between accessory and normal pathways. Morady et al described a very similar patient who appeared to have a nodoventricular pathway in association with a slow intranodal pathway. ${ }^{21}$ In their case, there was HV dissociation during preexcited tachycardia excluding intraventricular macroreentry. They argued that tachycardia reflected intranodal re-entry.

The combination of incidental nodoventricular conduction during atrioventricular re-entrant tachycardia involving a directaccessory pathway as in the patient in case 11 has also been described by Motte et $a^{22}$ and later by Lerman et al. ${ }^{23}$ In the latter report, the authors propose a macroreentry circuit comprising the nodoventricular pathway in the anterograde limb and a septal concealed accessory pathway in the retrograde limb. Nevertheless, the HV 
interval during tachycardia is $-25 \mathrm{~ms}$ suggesting anterograde activation of the His bundle and participation of both the normal and accessory pathways within the circuit with anterograde conduction in parallel over both pathways. The presence of varying fusion complexes at the onset of tachycardia without any associated changes in cycle length implies that the nodoventricular pathway is not on circuit.

Seven of the patients in this series had more than one type of tachycardia. In four patients at least two distinctly different mechanisms could be identified. In two, possibly three, patients a second accessory pathway was present. Multiple accessory pathways, although uncommon, are a well recognised cause of complex junctional re-entrant mechanisms. ${ }^{1024}$ Their presence is, however, often difficult to establish. Involvement of two such pathways in a tachycardia circuit excluding the atrioventricular node is rare, 7925 and if verapamil fails to affect tachycardia an erroneous diagnosis of ventricular tachycardia may be made.

In conclusion, broad complex tachycardias associated with ventricular pre-excitation may be caused by a multitude of different mechanisms and are often associated with complex junctional substrates-that is, more than one abnormal pathway. Pre-excitation during junctional tachycardia does not imply participation of an accessory pathway in the circuit. Several varieties of tachycardia mechanism may coexist and interact with each other. These arrhythmias may be misdiagnosed as ventricular tachycardia with consequent inappropriate investigations. The use of antiarrhythmic drugs with known effects (for example, verapamil, flecainide) is valuable in assessing these complex arrhythmias and should be considered during the electrophysiological study. Accurate diagnosis of the various diverse mechanisms is not possible without detailed studies, which may provide essential information on which to base treatment, whether it is medical, electrical, or surgical, or in particular, uses transvenous techniques designed to ablate accessory conduction. ${ }^{26}$ With regard to the last two approaches, determination of the role of the accessory pathway is essential.

We thank Dr A W Nathan for supplying details of the patient in case 10 .

\section{References}

1 Wellens HJJ, Durrer D. The role of an accessory atrioventricular pathway in reciprocal tachycardia. Observations in patients with and without the Wolff-Parkinson-White syndrome. Circulation 1975; 52: 58-72.

2 Ward DE, Camm AJ. Methodologic problems in the use of atrial pacing studies for the assessment of AV conduction. Clin Cardiol 1980; 3: 155-62.

3 Gallagher JJ, Pritchett ELC, Sealy WC, Kasell J, Wallace AG. The pre-excitation syndromes. Prog Cardiovasc Dis 1978; 20: 285-327.

4 Ward DE, Camm AJ, Spurrell RAJ. Ventricular pre-excitation due to anomalous nodo-ventricular pathways: report of three patients. Eur F Cardiol 1979; 9: 111-27.

5 Ward DE, Camm AJ, Cory-Pearce R, Fuenmayor I, Rees GM, Spurrell RAJ. Ebstein's anomaly in association with anomalous nodoventricular conduction. Preoperative and intraoperative electrophysiological studies. I Electrocardiol 1979; 12: 227-33.

6 Akhtar M. Electrophysiologic bases for wide QRS complex tachycardia. PACE 1983; 6: 81-98.

7 Wellens HJJ, Farre J, Bar FW. Wolff-Parkinson-White syndrome: value and limitations of programmed electrical stimulation. In Narula O, ed. Cardiac arrhythmias. Electrophysiology, diagnosis and management. Baltimore: Williams and Wilkins, 1979: 589-617.

8 Benditt DG, Pritchett ELC, Gallagher JJ. Spectrum of regular tachycardias with wide $Q R S$ complexes in patients with accessory atrioventricular pathways. Am $\mathcal{f}$ Cardiol 1978; 42: 828-38.

9 Kaku T, Fukatani M, Kiya F, Hashiba K. Wolff-ParkinsonWhite syndrome with bilateral accessory pathways both exhibiting antegrade and retrograde conduction. Am Heart $f$ 1981; 102: 296-9.

10 Pritchett ELC, Prystowsky EN, Benditt DG, Gallagher JJ. 'Dual atrioventricular nodal pathways' in patients with WolffParkinson-White syndrome. Br Heart $\mathcal{F}$ 1980; 43: 7-13.

11 Ward DE, Camm AJ, Spurrell RAJ. The response of regular reentrant supraventricular tachycardia to right heart stimulation. PACE 1979; 2: 586-95.

12 Touboul P, Vexler RM, Chatelain MT. Reentry via Mahaim fibres as a possible basis for tachycardia. Br Heart $\mathcal{f}$ 1978; 40: 806-11.

13 Gallagher JJ, Smith WM, Kasell JH, Benson DW Jr, Sterba R, Grant AO. Role of Mahaim fibres in cardiac arrhythmias in man. Circulation 1981; 64: 176-89.

14 Reiter MJ, Smith WM, Gallagher JJ. Clinical spectrum of ventricular tachycardia with left bundle branch morphology. Am $\mathcal{f}$ Cardiol 1983; 51:113-21.

15 Ko PT, Naccarelli GV, Gulamhusein S, Prystowsky EN, Zipes DP, Klein GJ. Atrioventricular dissociation during paroxysmal junctional tachycardia. PACE 1981; 4: 670-78.

16 Kuck KH, Brugada P, Freidman P, Wellens HJJ. Observations of the antidromic type of circus movement tachycardia in the Wolff-Parkinson-White syndrome. Foumal of the American College of Cardiology 1983; 2: 1003-10.

17 Trantham JL, Broughton A, Guarnieri T, German LD, Gallagher JJ. Two distinct V-H intervals in tachycardia associated with nodoventricular Mahaim fibres. Circulation 1982; 66 (suppl II): 269.

18 Sung RJ, Styperek JL. Electrophysiologic identification of dual atrioventricular nodal pathway conduction in patients with reciprocating tachycardia using anomalous bypass tracts. Circulation 1979; 60: 1464-76.

19 Weiss J, Cabeen WR Jr, Roberts NK. Nodoventricular accessory atrioventricular connection associated with dual atrioventricular pathways: a case report and review of the literature. $f$ Electrocardiol 1981; 14: 185-90.

20 Juma Z, Saksena S, Levy S, Wolff GS, Sung RJ. Electrophysiologic characteristics of nodoventricular bypass tract conduction in patients with dual atrioventricular nodal pathways [Abstract]. Circulation 1980; 62 (suppl III): 47.

21 Morady F, Scheinman MM, Gonzalez R, Hess D. His-ventricular dissociation in a patient with reciprocating tachycardia and a nodoventricular bypass tract. Circulation 1981; 64: 839-44.

22 Motte G, Brechenmacher C, Davy JM, Belhassen B. Assocation de fibres nodo-ventriculaires et atrio-ventriculaires à l'origine de tachycardies réciproques. Confrontation electro-physiologique et anatomo-pathologique. Arch Mal Coeur 1980; 73: 737-45.

23 Lerman BB, Waxman HL, Proclemer A, Josephson ME. Supraventricular tachycardia associated with nodoventricular and concealed atrioventricular bypass tracts. Am Heart $\mathcal{J}$ 1982; 104: 1097-102.

24 Gallagher JJ, Sealy W, Kasell J, Wallace AG. Multiple accessory pathways in patients with the pre-excitation syndrome. Circulation 1976; 54: 571-91.

25 Cinca J, Valle V, Gutierrez L, Figueras J, Ruis J. Reciprocating tachycardia using bilateral anomalous pathways: electrophysiologic and clinical implications. Circulation 1980; 62: 657-61.

26 Brodman R, Fisher J. Evaluation of a catheter technique for ablation of accessory pathways near the coronary sinus using a canine model. Circulation 1983; 67: 923-9. 